\title{
Implementation of CAPIO for Composite Adaptive Control of Cross-Coupled Unstable Aircraft
}

\author{
Yildiray Yildiz* \\ U. C. Santa Cruz, NASA Ames Research Center, Moffett Field, CA 94035, USA \\ Ilya V. Kolmanovsky ${ }^{\dagger}$ \\ University of Michigan, 1320 Beal Avenue Ann Arbor, MI 48109, USA
}

\begin{abstract}
This paper presents an implementation of a recently developed control allocation algorithm CAPIO (a Control Allocation technique to recover from Pilot Induced Oscillations) for composite adaptive control of an inertially cross coupled unstable aircraft. When actuators are rate-saturated due to either an aggressive pilot command, high gain of the flight control system or some anomaly in the system, the phase shift between the desired and the actual aircraft outputs and the effective time delay can increase. This effective time delay may degrade the performance or, depending on the severity of rate saturation, even destabilize the closed-loop system. The CAPIO reduces the effective time delay by minimizing the phase shift between the commanded and the actual attitude accelerations. While the original intent of CAPIO was to mitigate pilot induced oscillations, we have also found that CAPIO can help automatic flight controllers, in particular, adaptive flight controllers, avoid and recover from similar conditions caused by rate saturation effects. In the paper we report simulation results for a challenging scenario of an unstable aircraft with cross-coupling controlled by a composite adaptive controller in the presence of rate saturation. The simulations confirm the capability of CAPIO to serve as an effective rate saturation compensator in adverse conditions.
\end{abstract}

\section{Introduction}

Actuator rate saturation introduces an effective time delay (see Fig. 1) which, in general, degrades the system performance and can lead to system instability. In the case of aircraft, in particular, if the rate saturation persists, it can cause drastic degradation known as a Pilot Induced Oscillation (PIO). ${ }^{1}$ The authors' earlier work on CAPIO (a Control Allocation technique to recover from Pilot Induced Oscillations) ${ }^{2,3}$ addressed this problem by proposing a modified control allocation algorithm, which provided phase compensation to bring the desired and actual signals in-phase. Unlike previous approaches to PIO mitigation, CAPIO avoided the need for actuator ganging.

The CAPIO has two modes: Synchronization and tracking. In the synchronization mode, CAPIO minimizes the derivative of the error between the desired and the actual control signals, resulting in minimization of the phase shift. In the tracking mode, the error between the desired and the actual control signals is minimized, resulting in the convergence of the actual control signal to the desired control signal. Thanks to this dual behavior, phase minimization was achieved without any constant bias formation. It was assumed in the earlier work on $\mathrm{CAPIO}^{2}$ that there exists a PIO detector on board, which enables the switching between CAPIO modes depending on whether a PIO event has been detected.

In this paper, we demonstrate that CAPIO can be used as a general phase compensator to mitigate rate saturation effects regardless of the occurrence of the PIO event. This is achieved by simultaneous minimization of the error and of the derivative of the error which are combined into a cost function with appropriate weights.

*Associate scientist, University Affiliated Research Center, AIAA Member.

${ }^{\dagger}$ Professor, Department of Aerospace Engineering, AIAA Member. 
There are a handful of approaches for the input saturation problem in adaptive control. ${ }^{4-8}$ These approaches in general include the modification of the adaptive laws or the adaptive control signal itself to ensure the stability of the overall system in the presence of actuator saturation. In this paper we approach the problem from a control allocation standpoint where we employ our phase compensator CAPIO without altering the original adaptive controller. A control allocation approach has several advantages. First, one can separate the controller design and the control input distribution which enables reconfiguration without changing the controller design in the case of a failure or an objective change. ${ }^{9}$ Second, in the presence of redundant actuators, secondary objectives can be achieved along with a primary objective. For example, in flight control, drag profile shaping can be combined with trajectory following. For an introduction to control allocation, see Harkegard. ${ }^{9}$

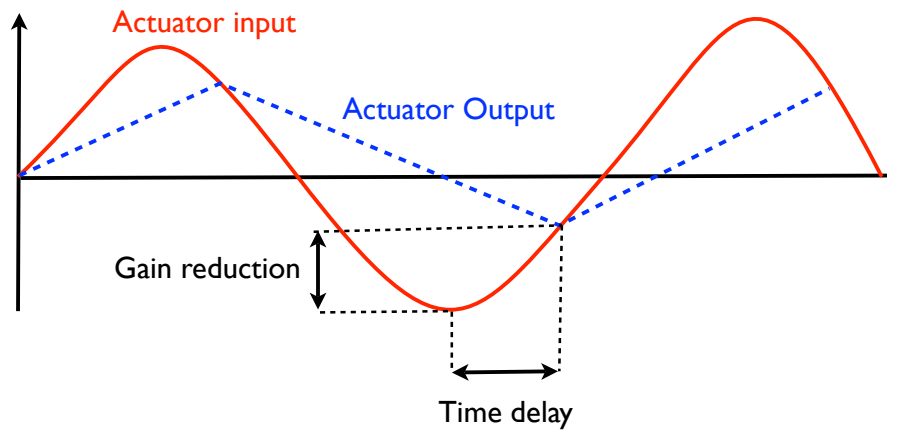

Figure 1. Input $u$ and output $\delta$ of a rate saturated actuator.

To illustrate the capabilities of CAPIO we use an unstable, inertially cross coupled aircraft model which is controlled by a combined/composite adaptive controller developed according to? ${ }^{\text {S See also }}{ }^{10-13}$ for related work on composite adaptive control. We demonstrate that CAPIO can help automatic flight controllers, in particular, adaptive flight controllers, avoid and recover from adverse conditions caused by rate saturation effects.

\section{System Model}

To show the advantages of CAPIO, a flight control example using a simplified ${ }^{14}$ ADMIRE model $^{15,16}$ is used with some modifications to simulate inertial cross coupling.

The linearized aircraft model at Mach 0.22 , altitude $3000 \mathrm{~m}$ is given by

$$
\begin{aligned}
& x=\left[\begin{array}{lllll}
\alpha & \beta & p & q & r
\end{array}\right]^{T}-x_{\text {lin }}, \\
& y=C x=\left[\begin{array}{lll}
p & q & r
\end{array}\right]^{T}-y_{\text {lin }}, \\
& \delta=\left[\begin{array}{llll}
\delta_{c} & \delta_{r e} & \delta_{l e} & \delta_{r}
\end{array}\right]^{T}-\delta_{\mathrm{lin}}, \\
& u=\left[\begin{array}{llll}
u_{c} & u_{\mathrm{re}} & u_{l e} & u_{r}
\end{array}\right]^{T}-u_{\text {lin }} \\
& {\left[\begin{array}{c}
\dot{x} \\
\dot{\delta}
\end{array}\right]=\left[\begin{array}{cc}
A_{0} & B_{x} \\
0 & -B_{\delta}
\end{array}\right]\left[\begin{array}{l}
x \\
\delta
\end{array}\right]+\left[\begin{array}{c}
0 \\
B_{\delta}
\end{array}\right] u,}
\end{aligned}
$$

where $\alpha, \beta, p, q$ and $r$ are the angle of attack, sideslip angle, roll rate, pitch rate and yaw rate, respectively. The $\delta$ and $u$ represent the actual and the commanded control surface deflections, respectively. Control surfaces are canard wings, right and left elevons and the rudder. (.)lin refers to values at the operating points where the linearization was performed. The actuators have the following position and rate limits

$$
\begin{aligned}
& \delta_{c} \in[-55,25] \times \frac{\pi}{180} ; \quad \delta_{r e}, \delta_{l e}, \delta_{r} \in[-30,30] \times \frac{\pi}{180} \\
& \dot{\delta}_{c}, \dot{\delta}_{r e}, \dot{\delta}_{l e}, \dot{\delta}_{r} \in[-70,70] \times \frac{\pi}{180}
\end{aligned}
$$

and have first order dynamics with a time constant of 0.05 seconds. It is noted that the position limits given are the same as the ones given by Harkegard ${ }^{14}$ but here we also impose the rate limits to illustrate CAPIO benefits. 
To make this model suitable for control allocation implementation, the actuator dynamics are neglected and the control surfaces are viewed as pure moment generators with their influence on $\dot{\alpha}$ and $\dot{\beta}$ neglected. It is noted that the actuators dynamics are present during the simulations, i.e. they are neglected only during the control allocation algorithm derivation. These assumptions lead to the following approximate linear model

$$
\begin{aligned}
\dot{x} & =A x+B_{u} u=A x+B_{v} v \\
v & =B u,
\end{aligned}
$$

where

$$
\begin{aligned}
& B_{u}=B_{v} B, \quad B_{v}=\left[\begin{array}{c}
0_{2 \times 3} \\
I_{3 \times 3}
\end{array}\right], \\
& A=\left[\begin{array}{ccccc}
-0.5432 & 0.0137 & 0 & 0.9778 & 0 \\
0 & -0.1179 & 0.2215 & 0 & -0.9661 \\
0 & -10.5128 & -0.9967 & 0 & 0.6176 \\
2.6221 & -0.0030 & 0 & -0.5057 & 0 \\
0 & 0.7075 & -0.0939 & 0 & -0.2127
\end{array}\right], \\
& B=\left[\begin{array}{ccccc}
0 & -4.2423 & 4.2423 & 1.4871 \\
1.6532 & -1.2735 & -1.2735 & 0.0024 \\
0 & -0.2805 & 0.2805 & -0.8823
\end{array}\right] .
\end{aligned}
$$

The virtual (total) control effort, $v$, consists of the angular accelerations in roll, pitch and yaw. To simulate the effects of inertial cross-coupling, we modify the $A$ matrix so that a change in pitch angular velocity creates a moment in roll and yaw axes:

$$
A=\left[\begin{array}{ccccc}
-0.5432 & 0.0137 & 0 & 0.9778 & 0 \\
0 & -0.1179 & 0.2215 & 0 & -0.9661 \\
0 & -10.5128 & -0.9967 & 1 & 0.6176 \\
2.6221 & -0.0030 & 0 & -0.5057 & 0 \\
0 & 0.7075 & -0.0939 & 0.1 & -0.2127
\end{array}\right]
$$

In this flight control example the goal of the inner loop adaptive controller is to regulate the roll, pitch and yaw angles at given reference values. The overall system configuration is given in Fig. 2.
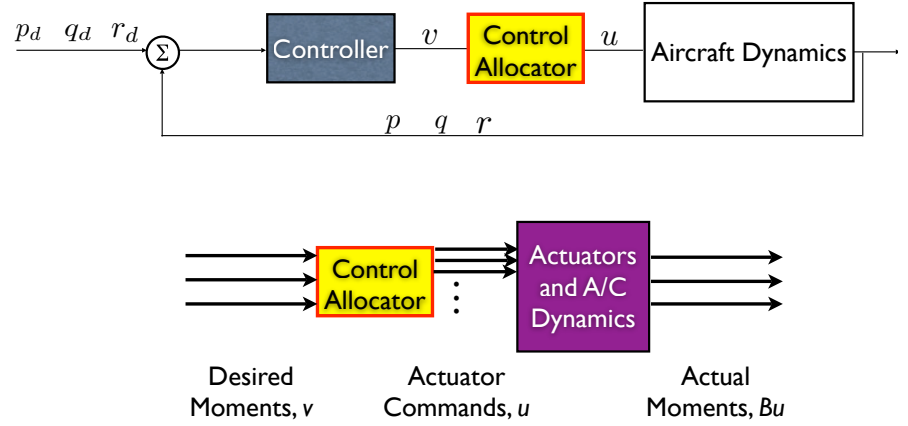

Figure 2. Overall MIMO system structure

The inner loop controller is a combined/composite adaptive controller, ${ }^{12}$ which uses $p_{d}, q_{d}$ and $r_{d}$ as references and produces the necessary attitude accelerations, $v \in \Re^{3}$, to track these references. The controller is designed assuming an input matched uncertainty model,

$$
\dot{x}=A x+B_{v}(v-\Phi(x, t) \theta)
$$


where $\Phi(x, t)$ is a known regressor matrix and $\theta$ is a vector of constant unknown parameters. The controller is given by

$$
v=\tilde{K} x+\tilde{L}\left[\begin{array}{c}
p_{d} \\
q_{d} \\
r_{d}
\end{array}\right]+\Phi(x, t) \hat{\theta},
$$

where $\tilde{K}$ and $\tilde{L}$ are appropriately defined matrices and where $\hat{\theta}$ is an adaptive feed-forward term which compensates for input matched uncertainties. The composite adaptation law is given by a parameter adjustment law, filters and gain adjustment law:

$$
\begin{gathered}
\dot{\hat{\theta}}=-\Gamma\left(e^{\mathrm{T}} P B_{v} \Phi(x, t)\right)^{\mathrm{T}}+a_{0} N^{\mathrm{T}}(e-\zeta), \\
\dot{\zeta}=-a_{0}(\zeta-e)+N(-\dot{\hat{\theta}})+A_{c l} e, \\
\dot{N}=-a_{0} N+B_{v} \Phi(x, t), \\
\lambda=\lambda_{0}\left(1-\frac{\|\Gamma\|}{k_{0}}\right), \\
\dot{\Gamma}=-a_{0} \Gamma N^{\mathrm{T}} N \Gamma+\lambda a_{0} \Gamma .
\end{gathered}
$$

In these equations $e=x-x_{e q}\left(p_{d}, q_{d}, r_{d}\right)$ denotes the tracking error, $A_{c l}=A+B_{v} \tilde{K}, a_{0}>0, \lambda_{0}>0$ and $k_{0}>0$. Note that the controller uses a time-varying gain matrix, $\Gamma$, and the adaptation is driven by both tracking error and estimation error to improve the convergence rate.

The control allocator distributes this total control effort, $v$, to individual control surfaces via the actuator commands, $u \in \Re^{4}$. The control surfaces then produce actual attitude accelerations, $B u$, where $B$ is the control input matrix.

\section{Simulation Results}

\section{III.A. Adaptive control with conventional control allocation}

The conventional control allocation used in this example minimizes the following objective function

$$
J=\|B u-v\|_{2}^{2}+\epsilon\|u\|_{2}^{2}
$$

subject to the constraints that $\max \left(\dot{u}_{\min } T+u_{k-1}, u_{\min }\right) \leq u \leq \min \left(\dot{u}_{\max } T+u_{k-1}, u_{\max }\right)$, where $T$ is the sampling interval. It is noted that norms, instead of square-norms, can be used in the objective function. Note that (5) is in the form of a typical objective function used in conventional control allocators, ${ }^{17}$ where the main objective is to minimize the error between the desired and the actual total control efforts. As $\epsilon \rightarrow 0$, minimizing (5) becomes equivalent to matching desired and actual attitude accelerations while picking the solution that gives the minimum control surface deflection, among different solutions. In our simulations, we used $\epsilon=10^{-5}$.

In the simulations, we assumed that $\Phi=I$, a $3 \times 3$ identity matrix. Figure 3 represents the adaptive controller performance when a conventional control allocator used. System goes into sustained oscillations due to the effective time delay introduced by rate saturating actuators (see Fig. 4).

\section{III.B. Adaptive control with CAPIO}

To minimize the phase shift and thus effective time delay due to rate saturation, CAPIO forces the virtual (total) control effort $v$, to be in phase with the actual control effort $B u$ produced by the actuators. To achieve this, a derivative error term is added to objective function (5) to obtain the following CAPIO objective function

$$
J^{\prime}=\|B u-v\|_{2}^{2}+\left\|W_{d}(B \dot{u}-\dot{v})\right\|_{2}^{2}+\epsilon\|u\|_{2}^{2},
$$

where $W_{d} \in R^{3 \times 3}$ represents a weighting matrix on the derivative term. The cost function $J^{\prime}$ is minimized with respect to $u$, with $\dot{u}=\left(u-u^{-}\right) / T$, where $u^{-}$denotes the value of $u$ at the previous sampling instant. It is noted that with this modified objective function, the control allocator is trying to realize $\dot{v}$ as well as $v$.

Figure 5 represents the same adaptive controller performance when CAPIO is used as the control allocator. Since CAPIO is able to minimize the phase shift between the commanded and achieved total control input vectors, the system is stable and the controller can successfully force the aircraft attitude angular rates follow their desired references. 

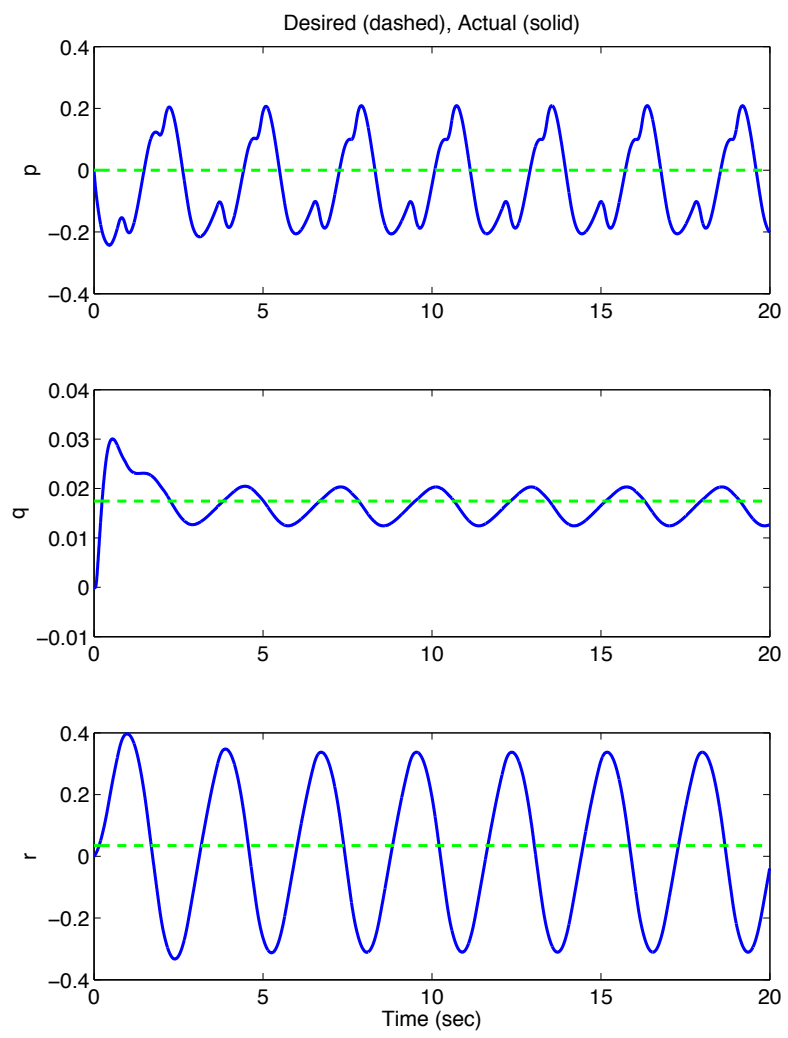

Figure 3. Regulation with adaptive controller + conventional control allocator

\section{Summary}

In this paper, we presented simulation results which demonstrate that the recently proposed control allocation scheme CAPIO can be used as an effective phase compensator for an adaptively controlled unstable aircraft with inertial cross-coupling in the presence of input saturation. CAPIO mitigates the rate saturation effect by its dual-behavior: It minimizes both the errors and the derivatives of the errors between the commanded and achieved total control signals. By minimizing the derivative errors, CAPIO prevents effective delay build-up, and by minimizing the errors themselves, CAPIO helps realizing the commanded control signals.

\section{Acknowledgments}

The authors gratefully acknowledge Diana Acosta, Susan Frost and Kalmanje Krishnakumar of NASA Ames Research Center, Jonathan Barlow of SGT Inc., and Gordon Hardy and Emily Lewis of SAIC. for helpful discussions.

\section{References}

${ }^{1}$ Klyde, D. H. and Mitchell, D. G., "Investigating The Role of Rate Limiting In Pilot-Induced Oscillations," Proc. AIAA Atmospheric Flight Mechanics Conference and Exhibit, AIAA Paper 2003-5463, Austin, Texas, Aug. 2003.

${ }^{2}$ Yildiz, Y. and Kolmanovsky, I. V., "A Control Allocation Technique to Recover From Pilot-Induced Oscillations (CAPIO) due to Actuator Rate Limiting," Proc. Amer. Control Conf., Baltimore, MD, 2010, pp. 516-523.

${ }^{3}$ Yildiz, Y. and Kolmanovsky, I. V., "Stability properties and cross coupling performance of the control allocation scheme CAPIO," Proc. AIAA Infotech@Aerospace, AIAA Paper 2010-3474, Ann Arbor, MI, 2010.

${ }^{4}$ Abramovitch, D. Y., Kosut, R. L., and Franklin, G. F., "Adaptive control with saturating inputs," Proc. Conference on Decision and Control, 1986, p. 848852. 

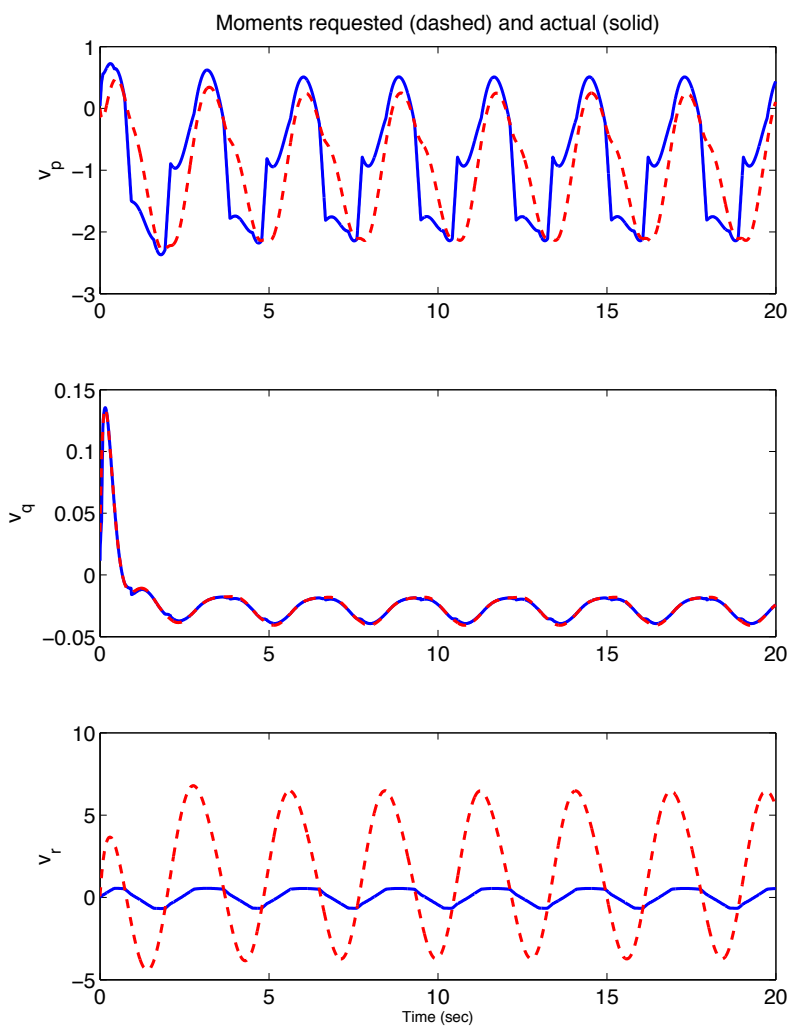

Figure 4. Requested and actual control inputs with adaptive controller + conventional control allocator

${ }^{5}$ Karason, S. P. and Annaswamy, A. M., "Adaptive Control in the Presence of Input Constraints," IEEE Trans. Automatic Control, Vol. 39, No. 11, Nov. 1994, pp. 2325-2330.

${ }^{6}$ Annaswamy, A. M. and Karason, S. P., "Discrete-time adaptive control in the presence of input constraints," Automatica, Vol. 31, 1995, pp. 14211431.

${ }^{7}$ Lavretsky E., Hovakimyan, N., "Stable adaptation in the presence of input constraints," Systems and Control Letters, Vol. 56, 2007, pp. 722-729.

${ }^{8}$ Leonessa, A., Haddad, W. M., Hayakawa, T., and Morel, Y., "Adaptive control for nonlinear uncertain systems with actuator amplitude and rate saturation constraints," Int. J. Adapt. Control Signal Process., Vol. 23, No. 1, 2009, pp. 73-96.

${ }^{9}$ Harkegard, O., Backstepping and Control Allocation with Applications to Flight Control, Ph.D. thesis, Linkoping University, 2003.

${ }^{10}$ Duarte, M. and Narendra, K., "Combined direct and indirect approach to adaptive control," Technical Report 8711 , Center for Syst. Sci., Yale Univ., New Haven, CT, Sep. 1987.

${ }^{11}$ Slotine, J.-J. E. and Li, W., "Composite adaptive control of robot manipulators," Automatica, Vol. 25, No. 4, 1989, pp. 509-519.

${ }^{12}$ Stotsky, A., "Lyapunov design for convergence rate improvement in adaptive control," Int. J. Control, Vol. 57, No. 2, 1993, pp. 501-504.

${ }^{13}$ Lavretsky, E., "Combined / Composite Model Reference Adaptive Control," Proc. AIAA Guidance. Navigation, and Control Conference, AIAA Paper 2009-6065, Chicago, IL, 2009.

${ }^{14}$ Ola Harkegard, S. and Glad, T., "Resolving Actuator Redundancy - Optimal Control vs. Control Allocation," Automatica, Vol. 41, 2005, pp. 137-144.

${ }^{15}$ Aerodata Model in Research Environment (ADMIRE), version 3.4h., Swedish Defence Research Agency (FOI), URL: www.foi.se/admire, 2003.

${ }^{16}$ Backstrm, H., "Report on the usage of the Generic Aerodata Model," Technical report, Saab Aircraft AB, May 1997.

${ }^{17}$ Bodson, M., "Evaluation of Optimization Methods for Control Allocation," Proc. AIAA Guidance, Navigation, and Control Conference and Exhibit, AIAA Paper 2001-4223, Montreal, CA, Aug. 2001. 

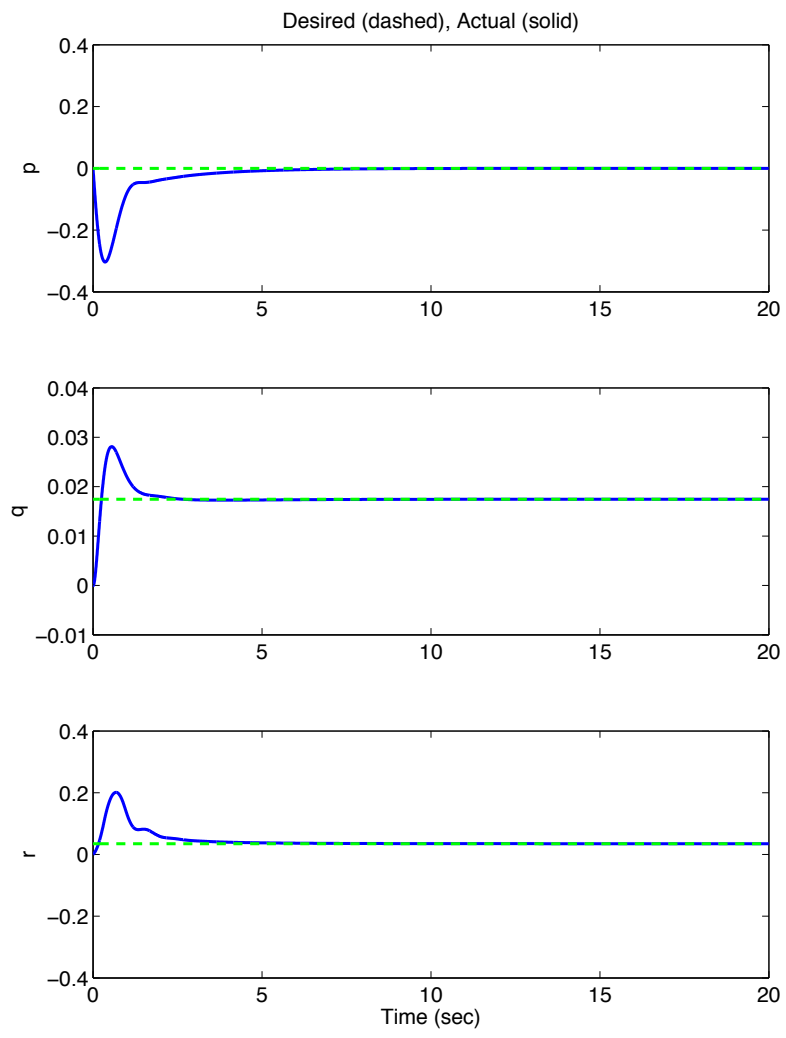

Figure 5. Regulation with adaptive controller + CAPIO 

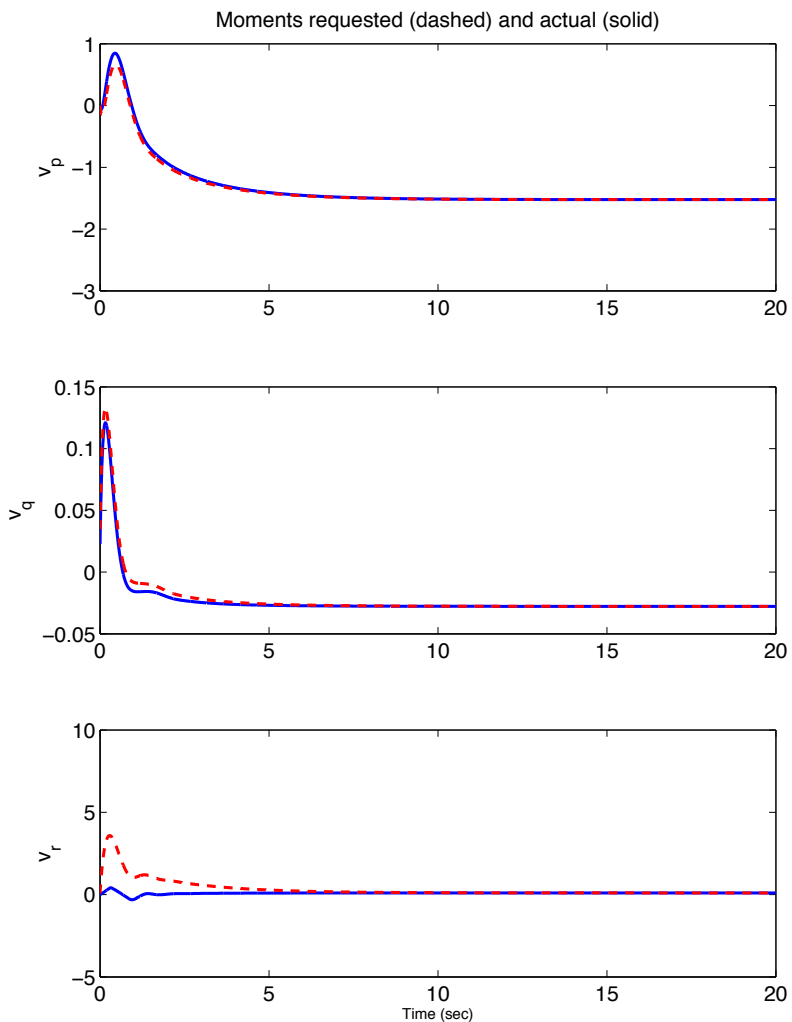

Figure 6. Requested and actual control inputs with adaptive controller + CAPIO 